\title{
The role of macrophage accumulation in adipose tissue in the development of obesity-induced insulin resistance
}

\author{
K. A. Harford ${ }^{1}$, M. Claessens ${ }^{1}$, E. Oliver ${ }^{1}$, K. H. G. Mills ${ }^{2}$ and H. M. Roche ${ }^{1}$ \\ ${ }^{1}$ Nutrigenomics Research Group, UCD Conway Institute, Belfield, University College Dublin, Republic of Ireland \\ and ${ }^{2}$ School of Biochemistry and Immunology, Trinity College Dublin, Republic of Ireland
}

\begin{abstract}
Macrophages are a heterogeneous population of cells that play a role in the innate immune response to infection. Recent studies have shown that macrophages are key cells in the development of obesity, wherein there is progressive infiltration of macrophages into the adipose tissue. These adipose tissue macrophages are referred to as classically-activated, or M1, macrophages ${ }^{(1)}$. They release proinflammatory cytokines such as IL-1, IL-6 and TNF $\alpha$, creating an inflammatory response that can contribute to insulin resistance and type 2 diabetes mellitus (T2DM) ${ }^{(2)}$. In lean individuals macrophages are in an M2-polarization or alternatively-activated state and are thought to protect against inflammation. The IL-1 receptor type 1 (IL-1R1) is responsible for transmitting the pro-inflammatory effects of IL-1. The aim of the present study was to determine the number of adipose tissue macrophages and their activation state using flow cytometry in control mice and in IL-1R1 $1^{-1-}$ mice at certain time points over a period of 12 weeks.

C57BL/6 control and IL-1R $1^{-1-}$ mice were fed a high-fat diet (45\% energy from fat) for 12 weeks. At week 6 epididymal adipose tissue samples were taken from control and IL-1R $1^{-/-}$mice. Adipocytes and stromal vascular cells (SVC) were isolated from the adipose tissue by collagenase treatment. SVC were labelled with antibodies for macrophage markers F4/80, CD11B and CD11C and analysed by flow cytometry to determine the number of macrophages and activation status of the macrophages present. Triple-positive cells

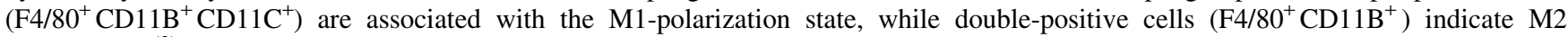
macrophages $^{(3)}$. Fasting glucose, insulin, triacylglycerol (TAG) and non-esterified fatty acid (NEFA) concentrations were measured at weeks 0 and 6 .

In the control group a larger population of the more active $\mathrm{F} 4 / 80^{+} \mathrm{CD} 11 \mathrm{~B}{ }^{+} \mathrm{CD} 11 \mathrm{C}^{+}$cells was present at week 6 compared with the IL-1R1 ${ }^{-/-}$group $(P<0.05)$. After 6 weeks on a high fat diet TG and NEFA levels were significantly higher in both control and IL-1R1 $1^{-/-}$groups. Fasting glucose concentrations did not differ significantly over time in either control or IL-1R $1^{-/-}$groups. However, insulin concentrations in the control group at week 6 were significantly higher when compared with week $0(P<0.05)$, pointing to insulin resistance. No significant differences in insulin levels in the IL-1R $1^{-/-}$mice were found.

These results imply that impairing IL-1 signalling decreases the macrophages ability to switch from an M2-polarization state to the proinflammatory M1-polarization state. This outcome reduces the infiltration of M1 macrophages into the adipose tissue, thereby lowering the pro-inflammatory response and attenuating the progression of insulin resistance. Further studies are continuing in order to determine the molecular mechanisms by which this process occurs.
\end{abstract}

This project is funded by the SFI Principal Investigator programme.

1. Lumeng CN, Bodzin JL \& Saltiel AR (2007) J Clin Invest 117, 175-184.

2. Weisburg SP, McCann D, Desai M, Rosenbaum M, Leibel RL \& Ferrante AW Jr (2003) J Clin Invest 112, 1796-1808

3. Nguyen AMT, Favelyukis S, Nguyen AK et al. (2007) J Biol Chem 282, 35279-35292. 